\title{
Association Between 16S rRNA Gene Mutations and Susceptibility to Amikacin in Mycobacterium avium Complex and Mycobacterium Abscessus Clinical Isolates
}

\author{
Su-Young Kim \\ Samsung Medical Center \\ Dae Hun Kim \\ Samsung Medical Center \\ Seong Mi Moon \\ Samsung Changwon Hospital, Sungkyunkwan University School of Medicine \\ Ju Yeun Song \\ Samsung Medical Center \\ Hee Jae Huh \\ Samsung Medical Center \\ Nam Yong Lee \\ Samsung Medical Center \\ Sung Jae Shin \\ Yonsei University \\ Won-Jung Koh \\ Samsung Medical Center \\ Byung Woo Jhun ( $\sim$ byungwoo.jhun@gmail.com ) \\ Samsung Medical Center
}

\section{Research Article}

Keywords: Mycobacterium avium complex, Mycobacterium abscessus, amikacin, drug resistance, rrs mutation

Posted Date: December 17th, 2020

DOI: https://doi.org/10.21203/rs.3.rs-127660/v1

License: (c) (1) This work is licensed under a Creative Commons Attribution 4.0 International License. Read Full License

Version of Record: A version of this preprint was published at Scientific Reports on March 17th, 2021. See the published version at https://doi.org/10.1038/s41598-021-85721-5. 


\section{Abstract}

We evaluated the association between 16S rRNA gene ( rrs) mutations and susceptibility in amikacin-resistant nontuberculous mycobacterial (NTM) clinical isolates in NTM- pulmonary disease (PD) patients. Susceptibility was retested for 134 amikacin-resistant isolates (minimum inhibitory concentration [MIC] $\geq 64 \mu \mathrm{g} / \mathrm{ml}$ ) from 86 patients. Amikacin resistance was reconfirmed in 102 NTM isolates from 62 patients with either Mycobacterium avium complex-PD (MAC-PD) ( $\mathrm{n}=54)$ or M. abscessus-PD ( $\mathrm{n=8}$ ). MIC and rrs mutations were evaluated for 318 single colonies from these isolates. For the 54 MAC-PD patients, rrs mutations were present in all isolates (obtained from 31 patients) with an amikacin MIC $\geq 128 \mu \mathrm{g} / \mathrm{ml}$, but only in a few isolates (from three of 23 patients) with an $\mathrm{MIC}=64 \mu \mathrm{g} / \mathrm{ml}$. For the eight $M$. abscessus-PD patients, all amikacin-resistant isolates (MIC $\geq 64 \mu \mathrm{g} / \mathrm{ml}) \mathrm{had} r \mathrm{rs}$ mutations. Of amikacin resistance isolates, $A 1408 \mathrm{G}$ mutation $(\mathrm{n}=29)$ was most common. Two novel mutations, C1496T and T1498A, were also identified. The culture conversion rate did not differ by amikacin MIC (20\% for $\mathrm{MIC}=64 \mu \mathrm{g} / \mathrm{ml} v s .7 \%$ for MIC $\geq 128 \mu \mathrm{g} / \mathrm{ml}, \mathrm{p}=0.468)$. In conclusion, all amikacin-resistant $M$. abscessus isolates had $r r s$ mutations, but in MAC isolates showing relatively low resistance $(\mathrm{MIC}=64 \mu \mathrm{g} / \mathrm{ml})$, rrs mutations were infrequently identified.

\section{Introduction}

Nontuberculous mycobacteria (NTM) are ubiquitous organisms that cause chronic disease, and the burdens of NTM-pulmonary disease (PD) are increasing globally, including in South Korea ${ }^{1,2}$. Among NTM species, Mycobacterium avium complex (MAC), which is mainly composed of $M$. avium and $M$. intracellulare, is the most common pathogen, and M. abscessus, predominantly composed of $M$. abscessus subsp. abscessus and $M$. abscessus subsp. massiliense, is the second most common pathogen in many countries ${ }^{3,4}$.

Amikacin is one of the most important parenteral antibiotics for treating NTM-PD, especially for MAC-PD and $M$. abscessus-PD ${ }^{5,6}$. For MAC-PD, amikacin is recommended for patients with advanced disease or those whose isolates acquired macrolide resistance ${ }^{7,8}$. Evidence from the CONVERT study indicates a benefit of adding liposomal amikacin inhalation for refractory MAC-PD ${ }^{9}$. Recent guidelines from the American and British Thoracic Societies for treating M. abscessus-PD recommend a multidrug therapy, including intravenous amikacin, based on results of drug susceptibility testing (DST) ${ }^{7,8,10}$. A meta-analysis also showed that parenteral amikacin use was associated with $M$. abscessus-PD treatment success ${ }^{11}$. Unfortunately, treatment outcomes for NTM-PD are still unsatisfactory, and a substantial proportion of NTM-PD patients remain refractory to treatment ${ }^{12-15}$. Given that approximately $90 \%$ of MAC or $M$. abscessus clinical isolates have been reported to be amikacin susceptible ${ }^{16,17}$, amikacin is one of the few drugs that can be used as salvage therapy in refractory disease, and it plays an important role in long-term NTM-PD treatment.

However, despite its importance, studies of the mechanisms of resistance to amikacin are lacking. Acquired resistance to amikacin results from point mutations at position 1408 in the rrs gene, encoding $16 \mathrm{~S}$ rRNA ${ }^{18}$, and isolates with these mutations are mostly isolated from patients with extensive exposure to amikacin or related aminoglycosides ${ }^{19,20}$. However, there are limited data from NTM clinical isolates on the association between these mutations and amikacin susceptibility. Therefore, we aimed to elucidate mechanisms of amikacin resistance in amikacin-resistant MAC and M. abscessus clinical isolates obtained from NTM-PD patients. This study should generate greater recognition of the association between 16S rRNA gene mutations and susceptibility to amikacin in clinical NTM isolates. 


\section{Results}

DST for amikacin. A total of 134 reportedly amikacin-resistant (MIC $\geq 64 \mu \mathrm{g} / \mathrm{ml}$ ) NTM clinical isolates from 86 NTM-PD patients (one to six isolates per patient) were retested for amikacin susceptibility. After excluding 24 patients whose isolates were determined to be susceptible or to have only intermediate resistance to amikacin on repeat testing, 62 patients with MAC-PD $(n=54)$ or M. abscessus-PD $(n=8)$ were reconfirmed to have amikacinresistant NTM isolates. From these patients, 102 stored isolates were selected, and 318 single colonies from the stored isolates were analyzed (Fig. 1). Amikacin MICs for the single colonies are shown in Supplementary Table S1. If any of the single colonies obtained from an isolate had an amikacin MIC $\geq 64 \mu \mathrm{g} / \mathrm{ml}$, the isolate was considered resistant to amikacin.

Patient characteristics. The clinical characteristics of the 62 NTM-PD patients with amikacin-resistant isolates are shown in Table 1. The median age was 64 years (interquartile range [IQR], 53-70 years), and 28 (45\%) were female. All but four patients had a history of exposure to amikacin or other aminoglycoside prior to confirmation of amikacin resistance. The median exposure duration was 91 days (IQR, 28-172 days). Most patients (87\%) were infected with MAC, whereas eight (13\%) were infected with M. abscessus. 
Table 1

Clinical Characteristics of the 62 Patients with NTM-PD ${ }^{a}$

\begin{tabular}{|ll|}
\hline Characteristic & Total $(\mathbf{n}=\mathbf{6 2})$ \\
\hline Age, year & $64(53-70)$ \\
\hline Female & $28(45)$ \\
\hline Exposure to amikacin & $23(37)$ \\
Intravenous administration ${ }^{\text {b }}$ & $1(2)$ \\
\hline Inhaled administration & $34(55)$ \\
Other aminoglycoside (streptomycin, kanamycin) & $4(6)$ \\
Unknown & \\
\hline Duration of amikacin exposure (days) & $91(28-172)$ \\
\hline Etiology & $54(87)$ \\
MAC & $21 / 54$ \\
\hline M. avium & $32 / 54$ \\
M. intracellulare & $1 / 54$ \\
\hline M. chimaera & $8(13)$ \\
\hline M. abscessus & $5 / 8$ \\
M. abscessus subsp. abscessus & $3 / 8$ \\
& \\
\hline M. abscessus subsp. massiliense & \\
\hline NTM-PD: nontuberculous mycobacterial pulmonary disease; MAC: M. avium complex. \\
\hline Values are numbers (\%) or medians (interquartile ranges). \\
\hline
\end{tabular}

rrs mutations. Supplementary Table S1 shows the results of rrs sequence analysis of the 318 single colonies obtained from the 102 stored clinical isolates, which included $84 \mathrm{MAC}$ isolates and $18 \mathrm{M}$. abscessus isolates. There were 250 single colonies from the 84 MAC isolates, and of these, all 50 colonies with an MIC $\geq 128 \mu \mathrm{g} / \mathrm{ml}$ had rrs mutations. However, of the 134 single colonies with an amikacin MIC $=64 \mu \mathrm{g} / \mathrm{ml}$ only three colonies (from patients no. A-7, A-16, and A-19 in Supplementary Table S1) had rrs mutations. From the $18 \mathrm{M}$. abscessus isolates, there were 68 single colonies, and rrs mutations were present in all 42 of them that had an amikacin MIC $\geq 64 \mu \mathrm{g} / \mathrm{ml}$. 
None of the single colonies with susceptible or intermediate susceptibility to amikacin $(\leq 32 \mu \mathrm{g} / \mathrm{ml}) \mathrm{had} r \mathrm{rs}$ mutations.

Our analyses were summarized based on isolate susceptibility to amikacin and rrs mutations, using a single representative isolate for each patient (Table 2). For patients who had two or more stored isolates, if any isolate had an rrs mutation, that isolate was chosen as the representative isolate. Consequently, among the 54 patients with MAC-PD, there were 31 patients who had representative isolates with an amikacin MIC $\geq 128 \mu \mathrm{g} / \mathrm{ml}$, all of which had had rrs mutations. However, of the remaining 23 MAC-PD patients with isolates with an amikacin MIC = $64 \mu \mathrm{g} / \mathrm{ml}$, only three had representative isolates with rrs mutations. For the eight patients with $M$. abscessus-PD, all amikacin-resistant isolates had rrs mutations. Overall, 42 patients (34/54 with MAC-PD and 8/8 with M. abscessusPD) developed amikacin-resistant disease due to rrs mutations.

Table 2

Amikacin Susceptibility and rrs Mutations in MAC and M. abscessus Clinical Isolates

\begin{tabular}{|c|c|c|c|c|c|c|c|c|c|}
\hline \multirow[t]{2}{*}{ Species } & \multirow{2}{*}{$\begin{array}{l}\text { Amikacin } \\
\text { MIC } \\
(\mu \mathrm{g} / \mathrm{ml})\end{array}$} & \multirow{2}{*}{$\begin{array}{l}\text { No. of } \\
\text { isolates }\end{array}$} & \multicolumn{7}{|c|}{ No. of isolates with $r s$ mutation at the specified positions ${ }^{a}$} \\
\hline & & & None & A1408G & C1409T & G1491C & G1491T & C1496T & T1498A \\
\hline \multirow{4}{*}{$\begin{array}{l}\operatorname{MAC}(n= \\
54)\end{array}$} & 64 & 23 & 20 & 1 & 0 & 1 & 0 & 1 & 0 \\
\hline & 128 & 5 & 0 & 3 & 0 & 1 & 0 & 1 & 0 \\
\hline & 256 & 3 & 0 & 0 & 2 & 0 & 1 & 0 & 0 \\
\hline & $>256$ & 23 & 0 & 21 & 0 & 1 & 1 & 0 & 0 \\
\hline \multirow{4}{*}{$\begin{array}{l}\text { M. } \\
\text { abscessus } \\
(\mathrm{n}=8)\end{array}$} & 64 & 3 & 0 & 0 & 3 & 0 & 0 & 0 & 0 \\
\hline & 128 & 0 & 0 & 0 & 0 & 0 & 0 & 0 & 0 \\
\hline & 256 & 1 & 0 & 0 & 0 & 0 & 0 & 0 & 1 \\
\hline & $>256$ & 4 & 0 & 4 & 0 & 0 & 0 & 0 & 0 \\
\hline \multicolumn{2}{|l|}{ Total } & 62 & 20 & 29 & 5 & 3 & 2 & 2 & 1 \\
\hline \multicolumn{10}{|c|}{ MAC: M. avium complex; MIC: minimum inhibitory concentration. } \\
\hline \multicolumn{10}{|c|}{${ }^{\mathrm{a}}$ Escherichia coli numbering } \\
\hline
\end{tabular}

Of the 42 representative isolates with rrs mutations, 29 (69\%) harbored a mutation of $A$ to $G$ at position 1408 (A1408G). Five had a C1409T mutation, three had a G1491C mutation, and two had a G1491T mutation. Two novel mutations were identified; two M. avium isolates had a C1496T mutation and one M. abscessus subsp. abscessus isolate had a T1498A mutation. A secondary structure model indicating the location of the rrs mutations in the MAC and $M$. abscessus clinical isolates is shown in Fig. 2.

Clinical outcomes of NTM-PD patients according to the MIC of amikacin. Overall, of the 62 study patients, 19 (31\%) (13 with $M$. avium-PD and six with $M$. intracellularePD) achieved culture conversion, and the median time from treatment to culture conversion was 104 days (IQR, 35-270 days). Additionally, this culture conversion was achieved in 10 of 26 patients (39\%) with MAC isolates with an amikacin MIC $=64 \mu \mathrm{g} / \mathrm{ml}$ compared to nine of 36 patients $(25 \%)$ with MAC isolates with an MIC $\geq 128 \mu \mathrm{g} / \mathrm{ml}(\mathrm{p}=0.279)$. No patients with $M$. abscessus-PD achieved culture conversion. 
For the 19 patients (nine M. aviumPD, five M. intracellulare-PD, three M. abscessus subsp. abscessus-PD, and two M. abscessus subsp. massiliensePD) who maintained amikacin therapy for $\geq 28$ days (median 256 days, IQR 60516 days) even after amikacin resistance was confirmed, the clinical outcome was re-analyzed. Among them, one patient with $M$. intracellularePD, out of $5(20 \%)$ patients who had NTM isolates with an MIC $=64 \mu \mathrm{g} / \mathrm{ml}$, achieved culture conversion, and one patient with M. avium-PD, out of 14 (7\%) patients who had NTM isolates with MIC $\geq$ $128 \mu \mathrm{g} / \mathrm{ml}$, achieved culture conversion $(\mathrm{p}=0.468)$. There was no statistical significance in the culture conversion rate according to amikacin MIC.

\section{Discussion}

In this study, we showed that mutations in the 16S rRNA gene of NTM clinical isolates are associated with amikacin resistance, and we also identified two novel amikacin resistance-associated mutations. Notably, all amikacinresistant $M$. abscessus isolates had rrs mutations. In MAC isolates, all isolates showing relatively high resistance $(\mathrm{MIC} \geq 128 \mu \mathrm{g} / \mathrm{ml})$ had $r \mathrm{rs}$ mutations, but only $13 \%(3 / 23)$ of isolates showing relatively low resistance $(\mathrm{MIC}=$ $64 \mu \mathrm{g} / \mathrm{ml}$ ) had rrs mutations. Additionally, among patients who maintained amikacin therapy, the culture conversion rate was low in the relatively high MIC group, although not significantly so $(20 \%$ in $\mathrm{MIC}=64 \mu \mathrm{g} / \mathrm{ml} v s$. $7 \%$ in $\mathrm{MIC} \geq$ $128 \mu \mathrm{g} / \mathrm{ml}, \mathrm{p}=0.468)$. However, a larger study with more patients may determine whether there are indeed significant differences between these two groups.

During amikacin therapy for NTM-PD, a susceptible strain can develop resistance. The mechanism of resistance in MAC isolates with high-level resistance and in $M$. abscessus isolates appears to be via rrs mutations, with the A1408G mutation being most common. The rrs mutations A1408G, C1409T, and G1491T had previously been found in kanamycin-resistant $M$. tuberculosis clinical isolates ${ }^{21}$, and these three mutations were also found in aminoglycoside-resistant $M$. abscessus subsp. abscessus mutants selected in vitro ${ }^{22}$. To our knowledge, ours is the first study to identify the G1491T mutation in MAC clinical isolates. Indeed, the mutation at G1491C and the additional two novel mutations, at C1496T and T1498A, which are also potentially involved in amikacin resistance, were first identified in mycobacterial isolates (Fig. 2). Based on our results, mechanisms of high resistance to amikacin involve rrs mutations.

However, these above-mentioned mutations were not present in all of our amikacin-resistant MAC isolates, and other mechanisms may contribute to low-level amikacin-resistance. In mycobacteria, aminoglycoside acetyltransferases are associated with low-level aminoglycoside resistance. The aminoglycoside acetyltransferase gene $\operatorname{aac}\left(2^{\prime}\right)$ was identified as a determinant of weak aminoglycoside resistance in $M$. fortuitum ${ }^{23}$. Also, the enhanced intracellular survival (Eis) protein, an aminoglycoside acetyltransferase, which was originally identified as a virulence factor in $M$. tuberculosis, confers kanamycin resistance ${ }^{24,25}$. In $M$. tuberculosis clinical isolates, rrs mutations correlated with high-level amikacin and kanamycin resistance, and eis promoter mutations correlated with low-level amikacin and moderate-level kanamycin resistance ${ }^{26}$. In another study with multidrug-resistant $M$. tuberculosis isolates, rrs mutations were associated with high-level cross-resistance to amikacin and kanamycin, whereas eis promoter mutations were associated with kanamycin resistance but not amikacin resistance 27. Aminoglycoside acetyltransferase activity could not be detected in MAC lysates in another study 28 , and thus, the role of these enzymes in MAC amikacin resistance is uncertain. Another possible drug-resistance mechanism is the drug efflux pump. The multidrug-resistant $M$. tuberculosis clinical isolate $M 7$, which was additionally resistant to amikacin, had no rrs mutations but overexpressed $p s t B$, an efflux pump gene ${ }^{29}$. Two efflux pump genes were also overexpressed in the amikacin- and kanamycin-resistant $M$. tuberculosis clinical isolate MT433, which lacked 
mutations, but overexpression of these genes in M. tuberculosis H37Ra strain did not increase the MIC against amikacin ${ }^{30}$. However, overexpression of eis in M. tuberculosis isolate MT433 was detected, suggesting that eis might be associated with kanamycin resistance ${ }^{30}$. There are limited studies about the basis for low-level amikacin resistance in $M$. tuberculosis, and further studies are also needed to understand the mechanism of low-level amikacin resistance in MAC.

In the process of selecting research subjects for our study, we identified cases where the amikacin MIC value in NTM clinical isolates changed across repeated measurements. The proportion of resistant isolates with both initial and repeated amikacin MICs $\geq 64 \mu \mathrm{g} / \mathrm{ml}$ was $72 \%$ (62/86, Fig. 1). Discrepancies in the amikacin MIC between initial and repeat testing of MAC isolates were also reported in a previous study; five of eight isolates with an initial amikacin $\mathrm{MIC}=64 \mu \mathrm{g} / \mathrm{ml}$ had an $\mathrm{MIC}=32 \mu \mathrm{g} / \mathrm{ml}$ on repeat testing, and seven of 10 isolates with an initial MIC > $64 \mu \mathrm{g} / \mathrm{ml}$ had reproducible results on repeat testing ${ }^{19}$. This phenomenon is thought to be due to polyclonal infection with strains with different amikacin MICs, but further studies are needed to elucidate any association. Nevertheless, repeat testing to confirm amikacin resistance may be helpful for determining therapeutic strategies for NTM-PD, especially MAC-PD.

In our study, amikacin MIC readings based on visible growth in the wells were uncertain for some MAC isolates, unlike for $M$. abscessus isolates. For MAC isolates with rrs mutations, all isolates had amikacin MICs $\geq 64 \mu \mathrm{g} / \mathrm{ml}$, and two-thirds $(26 / 34)$ had amikacin MICs $>256 \mu \mathrm{g} / \mathrm{ml}$. The growth of these isolates was evidenced by cell deposits at the bottom of the well (Supplementary Fig. S1B), and thus amikacin MIC interpretation was straightforward. In contrast, the cell deposits for MAC isolates without rrs mutations appeared to grow sporadically in wells at amikacin concentrations $\geq 64 \mu \mathrm{g} / \mathrm{ml}$ (Supplementary Fig. S1A). Because amikacin MIC interpretation for these cases was uncertain, growth in $64 \mu \mathrm{g} / \mathrm{ml}$ wells was confirmed by inoculating from MIC cultures into antimicrobial-free solid media. In this study, 46 MIC cultures showed non-confluent growth in the 64,128 , or $256 \mu \mathrm{g} / \mathrm{ml}$ wells, and thus the cultures in the $64 \mu \mathrm{g} / \mathrm{ml}$ wells were inoculated onto solid media and incubated; 12 grew on solid media whereas 34 showed no growth (final amikacin MIC = $64 \mu \mathrm{g} / \mathrm{ml}$ ). Therefore, unlike $M$. abscessus, interpretation of amikacin MICs in MAC isolates requires caution because some strains appear to grow sporadically. Repeat DST for amikacin and analysis for rrs mutations can be helpful for appropriate treatment.

From a clinical perspective, although it was not statistically significant, the negative culture conversion rate in our data tended to be slightly higher in patients with isolates with MIC $=64 \mu \mathrm{g} / \mathrm{ml}$ than in patients with MIC $\geq 128(20 \%$ vs. $7 \%$ ). According to the Clinical and Laboratory Standards Institute, $64 \mu \mathrm{g} / \mathrm{ml}$ represents a resistance breakpoint to amikacin, but there are no data on how helpful it would be to maintain amikacin therapy despite an MIC = $64 \mathrm{\mu g} / \mathrm{ml}$ in MAC-PD patients. We maintained amikacin in our study, even when amikacin resistance was confirmed, to obtain the synergistic effects with clofazimine or when resistance results were confirmed relatively late. In a recent CONVERT study that showed efficacy of liposomal amikacin inhalation for refractory MAC-PD, approximately $34 \%$ of patients $(10 / 29)$ having MAC isolates with an MIC $=64 \mu \mathrm{g} / \mathrm{ml}$ achieved culture conversion ${ }^{9}$, which suggests that amikacin may have some beneficial effects against MAC with low amikacin resistance; however, there is still no direct evidence for such benefits, and further studies on the association between MIC level and clinical outcomes are needed.

In conclusion, we identified several novel rrs mutations associated with amikacin resistance. In MAC isolates with relatively low resistance, rrs mutations were infrequently identified in contrast to $M$. abscessus isolates. Given the discrepancies between initial and re-tested susceptibility, repeat testing and/or rrs sequence analysis may be helpful for treating NTM-PD. 


\section{Methods}

Study population. The NTM Registry of the Samsung Medical Center in South Korea (ClinicalTrials.gov identifier: NCT00970801) was screened for patients with MAC- or M. abscessus-PD who had DST data obtained between October 2009 and June 2017 and which showed an amikacin minimum inhibitory concentration (MIC) $\geq 64 \mu \mathrm{g} / \mathrm{ml}$, resulting in a total of 101 patients. All patients fulfilled the diagnostic criteria for NTM-PD ${ }^{7}$ and were enrolled in an Institutional Review Board-approved observational cohort study investigating NTM-PD at Samsung Medical Center (approval no. 2008-09-016). Informed consent was obtained from all individual participants, and this study was approved by an Institutional Review Board at Samsung Medical Center. All methods were performed in accordance with the relevant guidelines and regulations. After excluding 15 patients without stored isolates, 86 NTM-PD patients were initially examined. A total of 134 amikacin-resistant NTM clinical isolates from the 86 NTM-PD patients (one to six isolates per patient) were retested for amikacin susceptibility. Twenty-four patients who had only susceptible or intermediately resistant isolates on repeat testing were further excluded (nine MAC and 15 M. abscessus). Finally, 62 patients (54 MAC and eight M. abscessus) whose isolates had MICs $\geq 64 \mu \mathrm{g} / \mathrm{ml}$ on both initial and repeat testing were included, and 102 isolates were obtained and analyzed (Fig. 1).

Microbiological examinations. In our hospital, processed specimens were inoculated into the BACTEC MGIT system (BD Diagnostics, Sparks, MD, USA). Liquid cultures were used for NTM identification and DST. NTM species were identified using PCR-restriction fragment length polymorphism analysis or reverse-blot hybridization of the $r p o B$ gene in routine clinical practice ${ }^{31}$. Beginning in June 2014, species identification was conducted via nested multiplex PCR and a reverse-hybridization assay of the internal transcribed spacer (ITS) region (AdvanSure ${ }^{\mathrm{TM}}$ Mycobacteria GenoBlot Assay; LG Life Sciences, Seoul, South Korea) ${ }^{32}$. DST for amikacin was initially performed at the Korean Institute of Tuberculosis using the broth microdilution method ${ }^{33}$. For research, NTM isolates from broth medium were first subcultured on $3 \%$ Ogawa agar before harvesting and storage at $-80{ }^{\circ} \mathrm{C}$.

From the 86 NTM-PD patients with available stored specimens (Fig. 1), a total of 134 NTM clinical isolates were propagated on Middlebrook 7H10 agar plates (Difco Laboratories, Detroit, MI, USA) supplemented with 10\% ( $\mathrm{vol} / \mathrm{vol}$ ) oleic acid-albumin-dextrose-catalase (OADC) (BD Diagnostics), and 425 single colonies were obtained. Three single colonies were routinely selected for each stored isolate or one to three single colonies of each morphotype when isolates included both smooth and rough morphotypes. Single colonies were re-identified using multilocus sequence analysis of $r r s, h s p 65$, the 16S-23S rRNA gene ITS, and $r p o B^{34,35}$ and were retested for amikacin susceptibility in the laboratory using the same method ${ }^{33}$. MAC and M. abscessus isolates were considered susceptible (MIC $\leq 16 \mu \mathrm{g} / \mathrm{ml}$ ), intermediate (MIC $=32 \mu \mathrm{g} / \mathrm{ml}$ ), or resistant (MIC $\geq 64 \mu \mathrm{g} / \mathrm{ml}$ ) to amikacin according to the Clinical Laboratory Standards Institute M62 protocol ${ }^{36}$. For MAC isolates with non-confluent growth at the bottom of the MIC-testing wells at amikacin concentrations of $\geq 64 \mu \mathrm{g} / \mathrm{ml}$, the growth in the $64 \mu \mathrm{g} / \mathrm{ml}$ wells was confirmed by inoculating from the MIC-testing cultures onto antimicrobial-free solid media (7H10 agar plates supplemented with OADC).

Treatment and clinical outcomes. All patients were treated with macrolide-based multidrug regimens ${ }^{37}$. In MAC-PD patients, a macrolide-based regimen that included ethambutol and rifampin was used. Aminoglycosides were administered in patients with severe disease. In M. abscessus-PD patients, an initial 2- to 4-week course of amikacin and imipenem (or cefoxitin) was administered during hospitalization, together with oral medications including a macrolide or fluoroquinolone, after which oral regimens were maintained. Some patients with refractory NTM-PD 
received oral clofazimine or inhaled amikacin. Sputum culture conversion was defined as three consecutive negative cultures, and the time to culture conversion was defined as the time to the date of the first negative culture.

rrs gene sequencing. Mycobacterial DNA was extracted using the DNeasy UltraClean Microbial Kit (Qiagen, Hilden, Germany) and was used as the template for PCR amplification of the last $351 \mathrm{bp}$ of the rrs gene, from positions 1154 to 1504, using primers rrs1-F (5'-ATG ACG TCA AGT CAT CAT GCC-3') and rrs1-R (5'-AGG TGA TCC AGC CGC ACC TTC-3') ${ }^{22}$. The PCR products were purified and sequenced using the rrs1-F primer. The rrs sequences from clinical isolates were compared against the GenBank database by BLAST analysis (http://www.ncbi.nlm.nih.gov) and were analyzed by the CLUSTAL-W multiple sequence alignment program ${ }^{38}$ against corresponding sequences of M. avium subsp. hominissuis 104, M. avium subsp. hominissuis TH135, M. intracellulare ATCC 13950, $M$. abscessus subsp. abscessus ATCC 19977, and M. abscessus subsp. massiliense JCM 15300.

Statistical analysis. Data are presented as number (\%) for categorical variables and median (IQR) for continuous variables. Categorical variables were compared using the Pearson chi-square test or Fisher's exact test. Statistical analyses were performed using the PASW software program (ver. 18.0; SPSS Inc., Chicago, IL, USA).

\section{Declarations}

\section{Author contributions}

S.K., D.H.K., S.M.M., S.S.J., W.K. contributed to the concept of the study. S.K. and D.H.K. contributed to the laboratory experiments. All authors contributed to the data analysis, and interpretation of data, and drafting and revision of the report. All authors approved the final version.

\section{Acknowledgments}

We would like to express our deepest gratitude for all that Professor Won-Jung Koh did for this research. The first person who proposed this study was Won-Jung Koh. After helping to initiate, design, and organize this study, WonJung Koh passed away in August of 2019.

\section{Funding}

This work was supported by the Basic Science Research Program through the National Research Foundation of Korea funded by the Ministry of Education (NRF-2016R1A6A3A11930364 and NRF-2019R1I1A1A01041381 to S.-Y. K.).

\section{Potential conflicts of interest}

All authors report no potential conflicts. All authors have submitted the ICMJE Form for Disclosure of Potential Conflicts of Interest. Conflicts that the editors consider relevant to the content of the manuscript have been disclosed.

Conflict of interest: The authors have no conflicts of interest to declare

Take-home message: All amikacin-resistant Mycobacterium abscessus isolates had rrs mutations. In M. avium complex isolates, all isolates with high amikacin resistance (MIC $\geq 128 \mu \mathrm{g} / \mathrm{ml}$ ) had rrs mutations, but only $13 \%$ of isolates with low resistance $(\mathrm{MIC}=64 \mu \mathrm{g} / \mathrm{ml})$ had $r r s$ mutations. 


\section{References}

1. Lee, H., Myung, W., Koh, W. J., Moon, S. M. \& Jhun, B. W. Epidemiology of nontuberculous mycobacterial infection, South Korea, 2007-2016. Emerging infectious diseases25, 569-572, http://doi.org/10.3201/eid2503.181597 (2019).

2. Prevots, D. R. \& Marras, T. K. Epidemiology of human pulmonary infection with nontuberculous mycobacteria: a review. Clinics in chest medicine36, 13-34, http://doi.org/10.1016/j.ccm.2014.10.002 (2015).

3. Ko, R. E. et al. Changing epidemiology of nontuberculous mycobacterial lung diseases in a tertiary referral hospital in Korea between 2001 and 2015. Journal of Korean medical science33, e65, http://doi.org/10.3346/jkms.2018.33.e65 (2018).

4. Hoefsloot, W. et al. The geographic diversity of nontuberculous mycobacteria isolated from pulmonary samples: an NTM-NET collaborative study. The European respiratory journal42, 1604-1613, http://doi.org/10.1183/09031936.00149212 (2013).

5. van Ingen, J., Ferro, B. E., Hoefsloot, W., Boeree, M. J. \& van Soolingen, D. Drug treatment of pulmonary nontuberculous mycobacterial disease in HIV-negative patients: the evidence. Expert review of anti-infective therapy11, 1065-1077, http://doi.org/10.1586/14787210.2013.830413 (2013).

6. Kang, Y. A. \& Koh, W. J. Antibiotic treatment for nontuberculous mycobacterial lung disease. Expert review of respiratory medicine10, 557-568, http://doi.org/10.1586/17476348.2016.1165611 (2016).

7. Griffith, D. E. et al. An official ATS/IDSA statement: diagnosis, treatment, and prevention of nontuberculous mycobacterial diseases. American journal of respiratory and critical care medicine175, 367-416, http://doi.org/10.1164/rccm.200604-571ST (2007).

8. Daley, C. L. et al. Treatment of nontuberculous mycobacterial pulmonary disease: an official ATS/ERS/ESCMID/IDSA clinical practice guideline. Eur Respir J56, http://doi.org/10.1183/13993003.005352020 (2020).

9. Griffith, D. E. et al. Amikacin liposome inhalation suspension for treatment-refractory lung disease caused by Mycobacterium avium complex (CONVERT). A prospective, open-label, randomized Study. Am J Respir Crit Care Med198, 1559-1569, http://doi.org/10.1164/rccm.201807-13180C (2018).

10. Floto, R. A. et al. US Cystic Fibrosis Foundation and European Cystic Fibrosis Society consensus recommendations for the management of non-tuberculous mycobacteria in individuals with cystic fibrosis. Thorax71 Suppl 1, i1-i22, http://doi.org/10.1136/thoraxjnl-2015-207360 (2016).

11. Kwak, N. et al.Mycobacterium abscessus pulmonary disease: individual patient data meta-analysis. Eur Respir J54, http://doi.org/10.1183/13993003.01991-2018 (2019).

12. Koh, W. J. et al. Clinical significance of differentiation of Mycobacterium massiliense from Mycobacterium abscessus. Am J Respir Crit Care Med183, 405-410, http://doi.org/10.1164/rccm.201003-03950C (2011).

13. Lyu, J. et al. A shorter treatment duration may be sufficient for patients with Mycobacterium massiliense lung disease than with Mycobacterium abscessus lung disease. Respiratory medicine108, 1706-1712, http://doi.org/10.1016/j.rmed.2014.09.002 (2014).

14. Roux, A. L. et al. Comparing Mycobacterium massiliense and Mycobacterium abscessus lung infections in cystic fibrosis patients. Journal of cystic fibrosis : official journal of the European Cystic Fibrosis Society14, 6369, http://doi.org/10.1016/j.jcf.2014.07.004 (2015).

15. Kwak, N. et al. Treatment outcomes of Mycobacterium avium complex lung disease: A systematic review and meta-analysis. Clin Infect Dis65, 1077-1084, http://doi.org/10.1093/cid/cix517 (2017). 
16. Cho, E. H. et al. Differences in drug susceptibility pattern between Mycobacterium avium and Mycobacterium intracellulare isolated in respiratory specimens. J Infect Chemother24, 315-318,

http://doi.org/10.1016/j.jiac.2017.10.022 (2018).

17. Kim, S. Y. et al. The drug susceptibility profile and inducible resistance to macrolides of Mycobacterium abscessus and Mycobacterium massiliense in Korea. Diagn Microbiol Infect Dis81, 107-111, http://doi.org/10.1016/j.diagmicrobio.2014.10.007 (2015).

18. Prammananan, T. et al. A single $16 \mathrm{~S}$ ribosomal RNA substitution is responsible for resistance to amikacin and other 2-deoxystreptamine aminoglycosides in Mycobacterium abscessus and Mycobacterium chelonae. $J$ Infect Dis177, 1573-1581, http://doi.org/10.1086/515328 (1998).

19. Brown-Elliott, B. A. et al. In vitro activity of amikacin against isolates of Mycobacterium avium complex with proposed MIC breakpoints and finding of a 16S rRNA gene mutation in treated isolates. J Clin Microbio/51, 3389-3394, http://doi.org/10.1128/jcm.01612-13 (2013).

20. Daniel-Wayman, S. et al. Amikacin exposure and susceptibility of macrolide-resistant Mycobacterium abscessus. ERJ Open Res5, http://doi.org/10.1183/23120541.00154-2018 (2019).

21. Suzuki, Y. et al. Detection of kanamycin-resistant Mycobacterium tuberculosis by identifying mutations in the 16S rRNA gene. Journal of clinical microbiology36, 1220-1225 (1998).

22. Nessar, R., Reyrat, J. M., Murray, A. \& Gicquel, B. Genetic analysis of new 16 S rRNA mutations conferring aminoglycoside resistance in Mycobacterium abscessus. The Journal of antimicrobial chemotherapy66, 17191724, http://doi.org/10.1093/jac/dkr209 (2011).

23. Hull, S. I. et al. Presence of aminoglycoside acetyltransferase and plasmids in Mycobacterium fortuitum. Lack of correlation with intrinsic aminoglycoside resistance. Am Rev Respir Dis129, 614-618 (1984).

24. Sanz-Garcia, F. et al. Mycobacterial Aminoglycoside Acetyltransferases: A Little of Drug Resistance, and a Lot of Other Roles. Front Microbio/10, 46, http://doi.org/10.3389/fmicb.2019.00046 (2019).

25. Campbell, P. J. et al. Molecular detection of mutations associated with first- and second-line drug resistance compared with conventional drug susceptibility testing of Mycobacterium tuberculosis. Antimicrob Agents Chemother55, 2032-2041, http://doi.org/10.1128/AAC.01550-10 (2011).

26. Kambli, P. et al. Correlating rrs and eis promoter mutations in clinical isolates of Mycobacterium tuberculosis with phenotypic susceptibility levels to the second-line injectables. Int J Mycobacterio/5, 1-6, http://doi.org/10.1016/j.ijmyco.2015.09.001 (2016).

27. Chakravorty, S. et al. Genotypic susceptibility testing of Mycobacterium tuberculosis isolates for amikacin and kanamycin resistance by use of a rapid sloppy molecular beacon-based assay identifies more cases of lowlevel drug resistance than phenotypic Lowenstein-Jensen testing. Journal of clinical microbiology53, 43-51, http://doi.org/10.1128/JCM.02059-14 (2015).

28. Ho, II, Chan, C. Y. \& Cheng, A. F. Aminoglycoside resistance in Mycobacterium kansasii, Mycobacterium aviumM. intracellulare, and Mycobacterium fortuitum: are aminoglycoside-modifying enzymes responsible? Antimicrob Agents Chemother44, 39-42, http://doi.org/10.1128/aac.44.1.39-42.2000 (2000).

29. Oh, T. S. et al. RNA expression analysis of efflux pump genes in clinical isolates of multidrug-resistant and extensively drug-resistant Mycobacterium tuberculosis in South Korea. Infect Genet Evol49, 111-115, http://doi.org/10.1016/j.meegid.2017.01.002 (2017).

30. Sowajassatakul, A., Prammananan, T., Chaiprasert, A. \& Phunpruch, S. Overexpression of eis without a mutation in promoter region of amikacin- and kanamycin-resistant Mycobacterium tuberculosis clinical strain. 
Ann Clin Microbiol Antimicrob17, 33, http://doi.org/10.1186/s12941-018-0285-6 (2018).

31. Koh, W. J. et al. Outcomes of Mycobacterium avium complex lung disease based on clinical phenotype. Eur Respir J50, 1602503, http://doi.org/10.1183/13993003.02503-2016 (2017).

32. Yang, M. et al. Comparative evaluation of the AdvanSure Mycobacteria GenoBlot assay and the GenoType Mycobacterium CM/AS assay for the identification of non-tuberculous mycobacteria. J Med Microbio/65, 14221428, http://doi.org/10.1099/jmm.0.000376 (2016).

33. Clinical Laboratory Standards Institute. Susceptibility testing of mycobacteria, Nocardia spp., and other aerobic actinomycetes. 2nd ed. CLSI document No. M24-A2 (Clinical Laboratory Standards Institute, 2011).

34. Kim, S. Y. et al. Molecular analysis of clinical isolates previously diagnosed as Mycobacterium intracellulare reveals incidental findings of "Mycobacterium indicus pranii" genotypes in human lung infection. BMC infectious diseases15, 406, http://doi.org/10.1186/s12879-015-1140-4 (2015).

35. Kim, S. Y. et al. Distribution and clinical significance of Mycobacterium avium complex species isolated from respiratory specimens. Diagnostic microbiology and infectious disease88, 125-137,

http://doi.org/10.1016/j.diagmicrobio.2017.02.017 (2017).

36. Clinical Laboratory Standards Institute. Performance standards for susceptibility testing of mycobacteria, Nocardia spp., and other aerobic actinomycetes. 1st ed. CLSI document No. M62 (Clinical Laboratory Standards Institute, 2018).

37. Jhun, B. W. et al. Prognostic factors associated with long-term mortality in 1445 patients with nontuberculous mycobacterial pulmonary disease: a 15-year follow-up study. Eur Respir J55, http://doi.org/10.1183/13993003.00798-2019 (2020).

38. Larkin, M. A. et al. Clustal W and Clustal X version 2.0. Bioinformatics23, 2947-2948, http://doi.org/10.1093/bioinformatics/btm404 (2007).

\section{Figures}

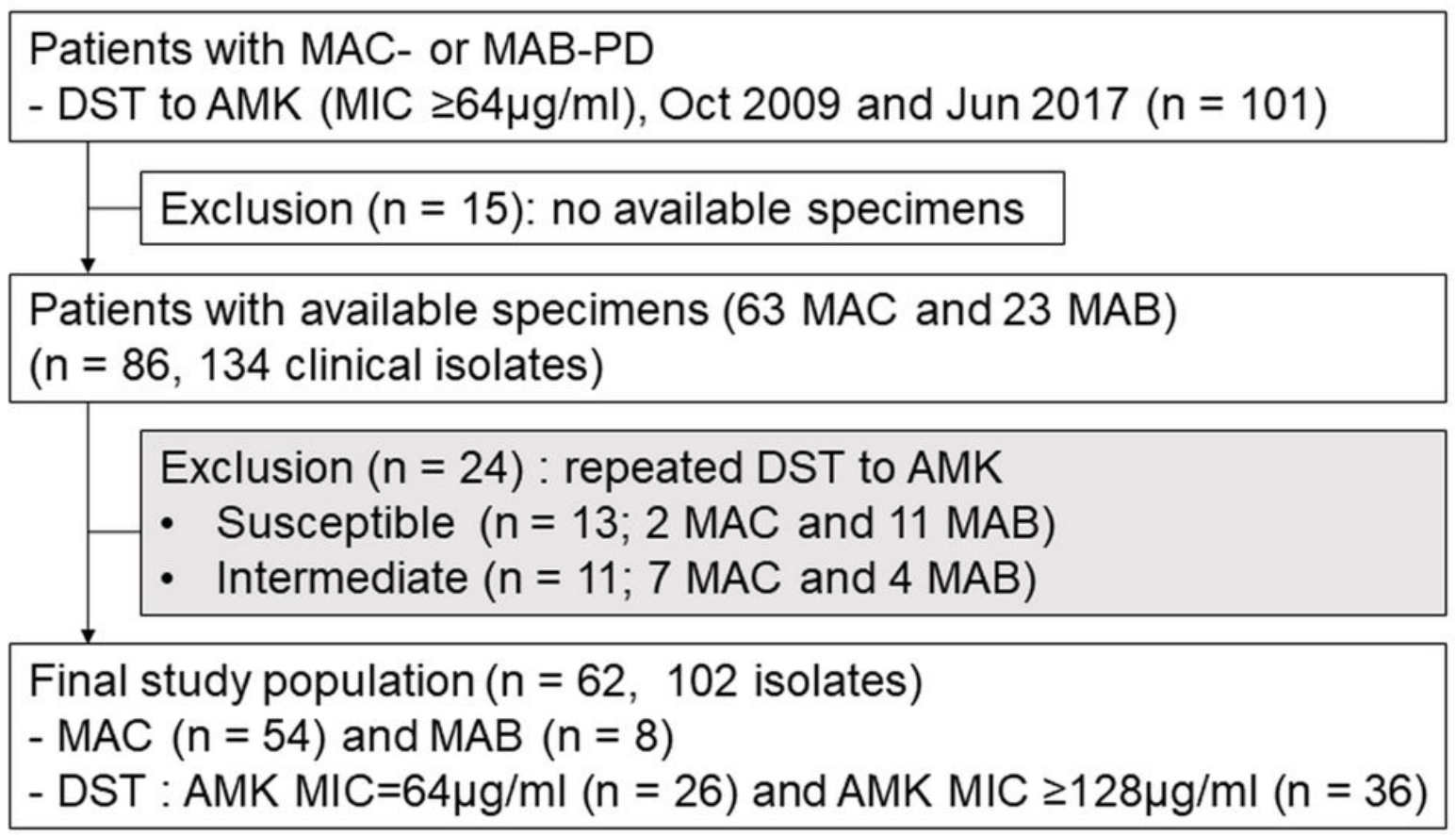


Figure 1

Study population. Abbreviations: MAC, M. avium complex; MAB-PD, M. abscessus-pulmonary disease; DST, drug susceptibility testing; AMK, amikacin; MIC, minimum inhibitory concentration.

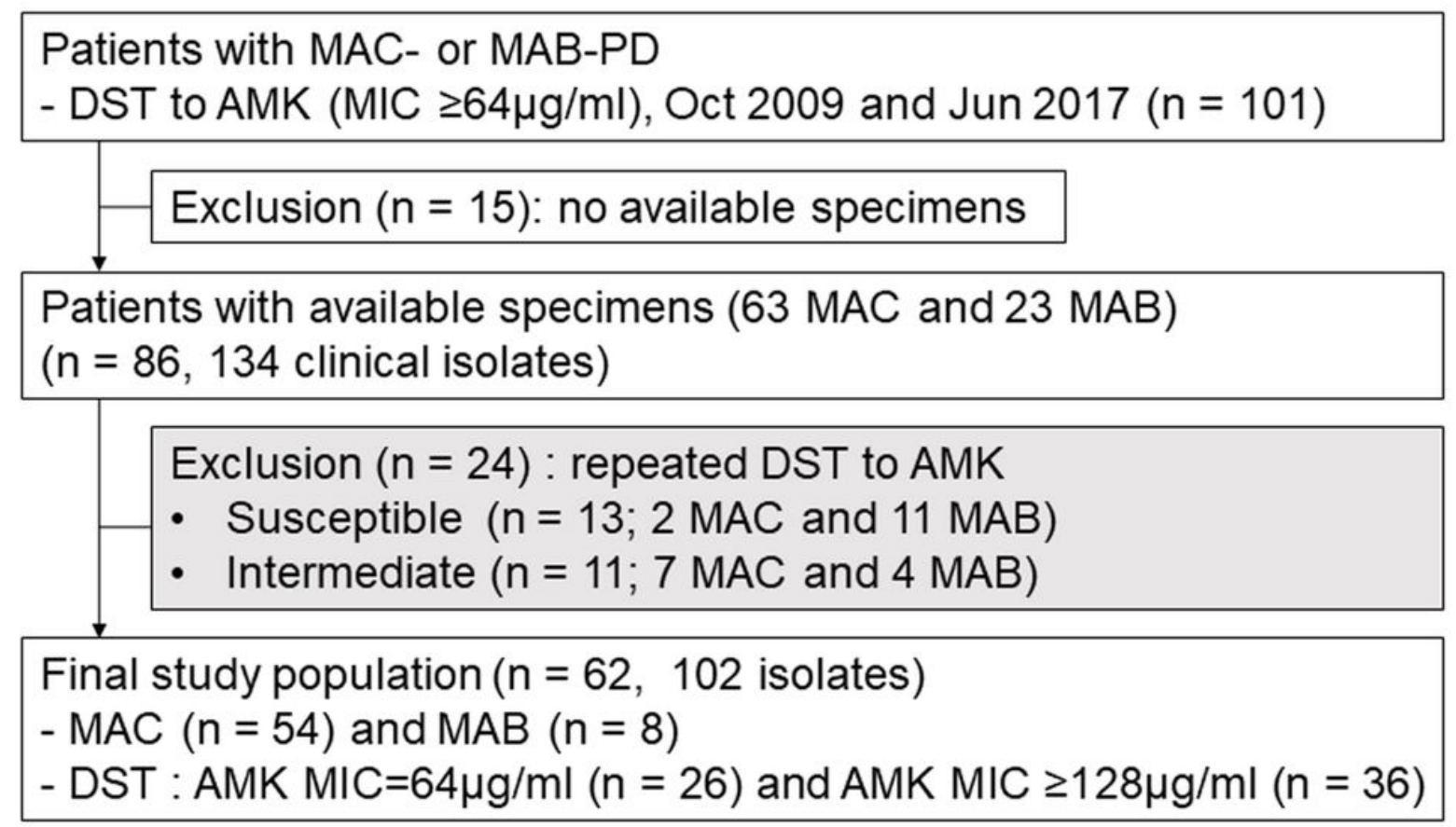

Figure 1

Study population. Abbreviations: MAC, M. avium complex; MAB-PD, M. abscessus-pulmonary disease; DST, drug susceptibility testing; AMK, amikacin; MIC, minimum inhibitory concentration.

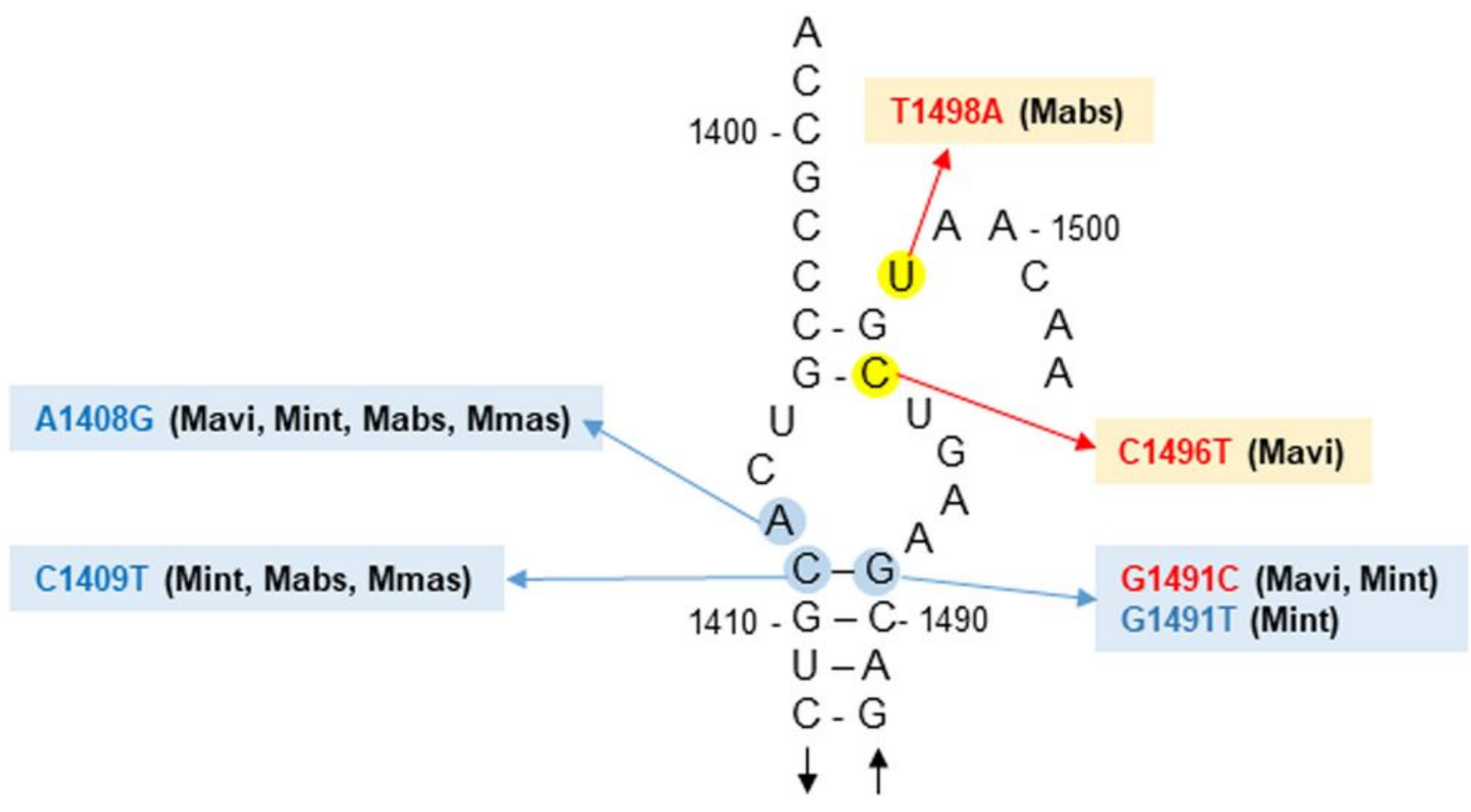




\section{Figure 2}

Locations of rrs mutations in amikacin-resistant MAC and M. abscessus clinical isolates. The species containing a given mutation are indicated with three-letter abbreviations: Mavi, M. avium; Mint, M. intracellulare; Mabs, M. abscessus subsp. abscessus; and Mmas, M. abscessus subsp. massiliense. E. coli numbering is used for the mycobacterial rrs sequence, and nucleotide positions at which mutations confer amikacin resistance are marked with blue or yellow circles. Blue circles indicate rrs mutations previously found in $\mathrm{M}$. tuberculosis clinical isolates and M. abscessus subsp. abscessus mutants selected in vitro, as well as in this study. Yellow circles indicate novel mutations identified in this study.

\section{Supplementary Files}

This is a list of supplementary files associated with this preprint. Click to download.

- SupplementaryFig.S1.tif

- SupplementaryFig.S1.tif

- SupplementaryTableNTMAMKRscientificreportsNEW.docx

- SupplementaryTableNTMAMKRscientificreportsNEW.docx 